\title{
Identification of DNA methylation of SOX9 in cervical cancer using methylated-CpG island recovery assay
}

\author{
JIAN-HONG WU ${ }^{1}$, XUE-AI LIANG ${ }^{2}$, YU-MEI WU ${ }^{1}$, FENG-SHUANG LI ${ }^{1}$ and YIN-MEI DAI ${ }^{1}$ \\ ${ }^{1}$ Beijing Obstetrics and Gynecology Hospital, Capital Medical University, Beijing 100026; \\ ${ }^{2}$ Haidian Maternal and Child Health Hospital, Beijing 100080, P.R. China
}

Received July 11, 2012; Accepted September 6, 2012

DOI: 10.3892/or.2012.2077

\begin{abstract}
The aim of the present study was to identify novel methylation markers for cervical cancer screening and to test the clinical application of the most promising biomarker in cervical scrapings. Methylated-CpG island recovery assaybased microarray analysis was carried out on a discovery set consisting of cervical cancer tissue and normal cervical tissue to identify significantly hypermethylated genes. Five hundred and four $\mathrm{CpG}$ islands, corresponding to 378 genes, were differentially methylated between cervical cancer tissue and normal cervical tissue. Among them, 30 genes were significantly hypermethylated. Of the 30 genes, SOX9, PKLR and DLX4 were selected for further validation by direct bisulfite sequencing. The SOX9 gene revealed complete methylation in the cervical cancer tissue and complete non-methylation in the normal control tissue. A TaqMan-based real-time PCR assay was performed to detect the methylation levels of the SOX9 gene in 156 cervical scrapings, including 48 normal cervical scrapings, 30 scrapings with cervical intraepithelial neoplasia 1 (CIN1), 30 scrapings with CIN2-3 and 48 scrapings with squamous cell carcinoma (SCC). The methylation levels (methylation score) of the SOX9 gene increased significantly with the severity of cervical squamous lesions. The area under the receiver operating characteristic (ROC) curve (AUC) revealed that the methylation score of the SOX9 gene could be used to segregate SCC/CIN2-3 from CIN1/normal (AUC, $0.961 ; \mathrm{P}=0.000)$. At the optimal cut-off value, a sensitivity of $92.3 \%$ and a specificity of $89.7 \%$ were obtained. In conclusion,
\end{abstract}

Correspondence to: Professor Yin-Mei Dai, Beijing Obstetrics and Gynecology Hospital, Capital Medical University, 251 Yaojia Yuan Street, Chaoyang, Beijing 100026, P.R. China

E-mail: daiyinmei@yahoo.com.cn

Abbreviations: CIN, cervical intraepithelial neoplasia; SCC, squamous cell carcinoma; hr-HPV, high-risk human papillomavirus; SOX9, SRY (sex determining region Y)-box 9; MIRA, methylated-CpG island recovery assay

Key words: cervical cancer, cervical intraepithelial neoplasia, methylation, $\mathrm{SOX} 9$, microarray
SOX9 methylation is frequently involved in cervical carcinogenesis, and may provide a valuable molecular biomarker for early detection of cervical cancer.

\section{Introduction}

Cervical cancer is the 2nd most frequent gynecological malignancy among women in the world, with the highest incidence in developing countries (1). It is estimated that 490,000 new cervical cancer cases are diagnosed and 270,000 deaths occur annually worldwide (2). Cervical cancer has a long precancerous phase, spanning years. The precancerous phase includes cervical intraepithelial neoplasia 1 (CIN1), CIN2 and CIN3. Since the introduction of a cytology test for cervical cancer screening, the rates of mortality and morbidity for patients with invasive cervical cancer have significantly decreased, particularly in developing countries. Nevertheless, cytological screening is not an ideal method due to its low sensitivity of only $55 \%$ for high-grade cervical intraepithelial neoplasia (CIN2 and CIN3) (3). Cervical carcinogenesis is highly associated with high-risk human papillomavirus (hr-HPV) infection and hr-HPV testing has been shown to improve the sensitivity of cervical screening (4). However, one of the main problems of hr-HPV testing is the low specificity for the identification of CIN2 or worse lesions, especially in young women, which results in a high false-positive rate (5).

In current practice, most patients with CIN2 or CIN3 have surgical intervention (6). It is estimated that when CIN2 is left untreated $40-60 \%$ of the lesions regress, $20 \%$ progress to $\mathrm{CIN} 3$ and $5 \%$ progress to invasive cancer. For untreated CIN3, 30-50\% regress and $10-40 \%$ progress to invasive cancer $(7,8)$. For untreated CIN1, most lesions regress to normal and about $10 \%$ progress to CIN2 or CIN3 (9). For the majority of CIN1 patients, a watchful waiting approach is adopted. This dichotomy in clinical follow-up is based on the diagnostic accuracy of microscopic visual inspection and may result in either over- or under-treatment. The question arises as to whether new independent diagnostic methods could complement the traditional approach, prompting the need for the development of new biomarkers for cervical cancer screening. Since aberrant DNA methylation of tumor suppressor genes is a frequent occurrence in most human tumors and occurs early in neoplastic progression (10), DNA methylation biomarkers may have potential as cervical cancer screening markers. 
A growing number of methylated genes have been associated with different grades of cervical neoplasia $(11,12)$. Most investigations of DNA methylation in cervical cancer used candidate genes that were known to be methylated in other types of cancer, such as DAPK, MGMT, RAR $\beta$ and ADCYAP1 (13-16). However, only a few large-scale, wide-genome studies have been performed to identify possible methylation markers in cervical carcinogenesis (17-19). To date, due to the moderate sensitivity and specificity, no methylation marker can be readily translated for use in the screening of cervical cancer (12). In this study, a genome-wide approach was used to identify novel hypermethylated genes with better performance for cervical cancer screening.

The aims of the present study were: i) to identify novel methylation biomarkers in a discovery set of fresh frozen tissues through methylated-CpG island recovery assay (MIRA) microarray, and ii) to further validate the most promising methylation biomarker in a test set of cervical scrapings.

\section{Materials and methods}

Patients and clinical specimens. All samples were collected from patients at the Beijing Obstetrics and Gynecologic Hospital from 2010 to 2011. A discovery set of primary cervical squamous cell cancer tissue $(\mathrm{n}=1)$ and normal cervical tissue ( $\mathrm{n}=1)$ was obtained from patients undergoing surgery. The tissue samples were immediately frozen in liquid nitrogen and stored at $-80^{\circ} \mathrm{C}$ until nucleic acid extraction. A test set of 156 scrapings was collected in ThinPrep Cytology containers and was stored at $4^{\circ} \mathrm{C}$ until use, according to the manufacturer's protocol. Patients referred for cervical cancer or with an abnormal cervical smear were selected. Healthy women who visited the hospital for a routine cytology test during the same period were randomly invited as healthy controls. For all cervical cancer patients, an examination under general anesthesia was planned for staging in accordance with the International Federation of Gynecology and Obstetrics (FIGO) criteria. During the examination, samples were obtained by a gynecologist. These patients were divided into: 22 (46\%) FIGO stage IB, 3 (6\%) FIGO stage IIA, 15 (31\%) FIGO stage IIB, 1 (2\%) FIGO stage IIIA and 7 (15\%) FIGO stage IIIB. For patients referred with an abnormal cervical smear, samples were taken during routine gynecologic examinations at the first outpatient clinic visit. All the patients with CIN and squamous cell carcinoma (SCC) were confirmed by histological examination. A total of 156 women, including 48 healthy controls (median age, 36 years); 30 with CIN1 (median age, 35 years); 30 with CIN2-3 (median age, 38 years); and 48 with SCC (median age, 50 years), were enrolled in this study. All women provided written informed consent. Exclusion criteria were pregnancy, a history of previous radiotherapy or chemotherapy, the presence of other cancers and past uterine cervix surgery. The study was approved by the Ethics Committee of our hospital.

Screening of hypermethylated genes using MIRA microarray analysis. MIRA method was undertaken on a genome-wide scale in this study. The MIRA microarray analysis was carried out as described by Rauch and Pfeifer (20) with minor modifications. Briefly, $2 \mu \mathrm{g}$ genomic DNA samples, separated from fresh frozen cervical tissues using standard techniques, were digested with MseI (5'-TTAA). The fragmented DNA was eluted to remove fragments smaller than $100 \mathrm{bp}$ using a microDNA Purification kit (Beijing CoWin Biotech Co., Ltd., Beijing, China). Then, eluted DNA fragments were used to enrich the methylated DNA by a BioChain MBD kit according to the manufacturer's protocols. The MIRA-captured DNA segments were amplified using a GenomePlex Whole Genome Amplification kit (Sigma) according to the instructions of the manufacturer. The products of whole genome amplification from cervical cancer tissue and normal cervical tissue were labeled with either cy3-dCTP or cy5-dCTP using Klenow enzyme (Takara, Dalian, China). Then the fluorescent dye labeled DNA segments were pooled and hybridized to Agilent human $\mathrm{CpG}$ island microarrays, which contain $61,982 \mathrm{CpG}$ dinucleotides, covering 4,162 genes. To eliminate possible dye bias, the hybridizations were performed in duplicate with a dye swap. Scanning and washing were carried out on Agilent's Microarray platform according to Agilent's standard protocols. Array data were analyzed using Agilent Feature Extraction software. Following global mean normalization, probes with an intensity $<400$ were set aside for further analysis. Probes were selected as positive if their fold-changes were $>1.5$ in two dye-swap slides. For our analysis, genes with at least two positive probes were defined as significantly hypermethylated genes. The schematic diagram for our MIRA-assisted CpG island microarray analysis is outlined in Fig. 1. Among the several methods for analyzing DNA methylation patterns on a genome-wide scale, the MIRA method is not based on restriction endonucleases, antibodies or sodium bisulfite treatment of the genomic DNA, but on the ability of methyl-CpG binding protein MBD to specifically recognize methylated $\mathrm{CpG}$ dinucleotides and the ability of MIRA to work on normal double-stranded DNA. In addition, the MIRA enrichment method has proven to be compatible with microarray platforms.

Validation of hypermethylated genes by direct bisulfite sequencing. From the methylated genes identified by methylation microarray analysis, three significantly hypermethylated genes (SOX9, DLX4 and PKLR) which had not been previously associated with cervical cancer were selected for further validation using direct bisulfite sequencing in the DNA obtained from the above tissues. First, genomic DNA was modified with bisulfite using the DNA methylation detection kit (BioChain, Hayward, CA, USA). Then, $125 \mathrm{ng}$ of sodium bisulfite-treated DNA was used for a program of 45 cycles of melting $\left(30 \mathrm{sec}\right.$ at $94^{\circ} \mathrm{C}$ ), annealing ( $30 \mathrm{sec}$ at $\left.55^{\circ} \mathrm{C}\right)$ and extension $\left(30 \mathrm{sec}\right.$ at $\left.72^{\circ} \mathrm{C}\right)$ in $50 \mu 1$ reaction mixture containing 1X PCR Buffer ( $\mathrm{Mg}^{2+}$ Plus), $200 \mu \mathrm{M}$ of each dNTP, $0.5 \mu \mathrm{M}$ of forward primers, $0.5 \mu \mathrm{M}$ of reverse primers (Table I) and 1.25 units of Takara Taq HS. PCR products were then eluted using a microDNA Purification kit (Beijing CoWin Biotech Co., Ltd.). The eluted PCR products were directly subjected to sequencing using forward primers of PCR amplification.

Quantitative DNA methylation analysis by TaqMan-based real-time PCR. SOX9 was selected for further analysis based on the direct bisulfite-modified sequencing results. To deter- 


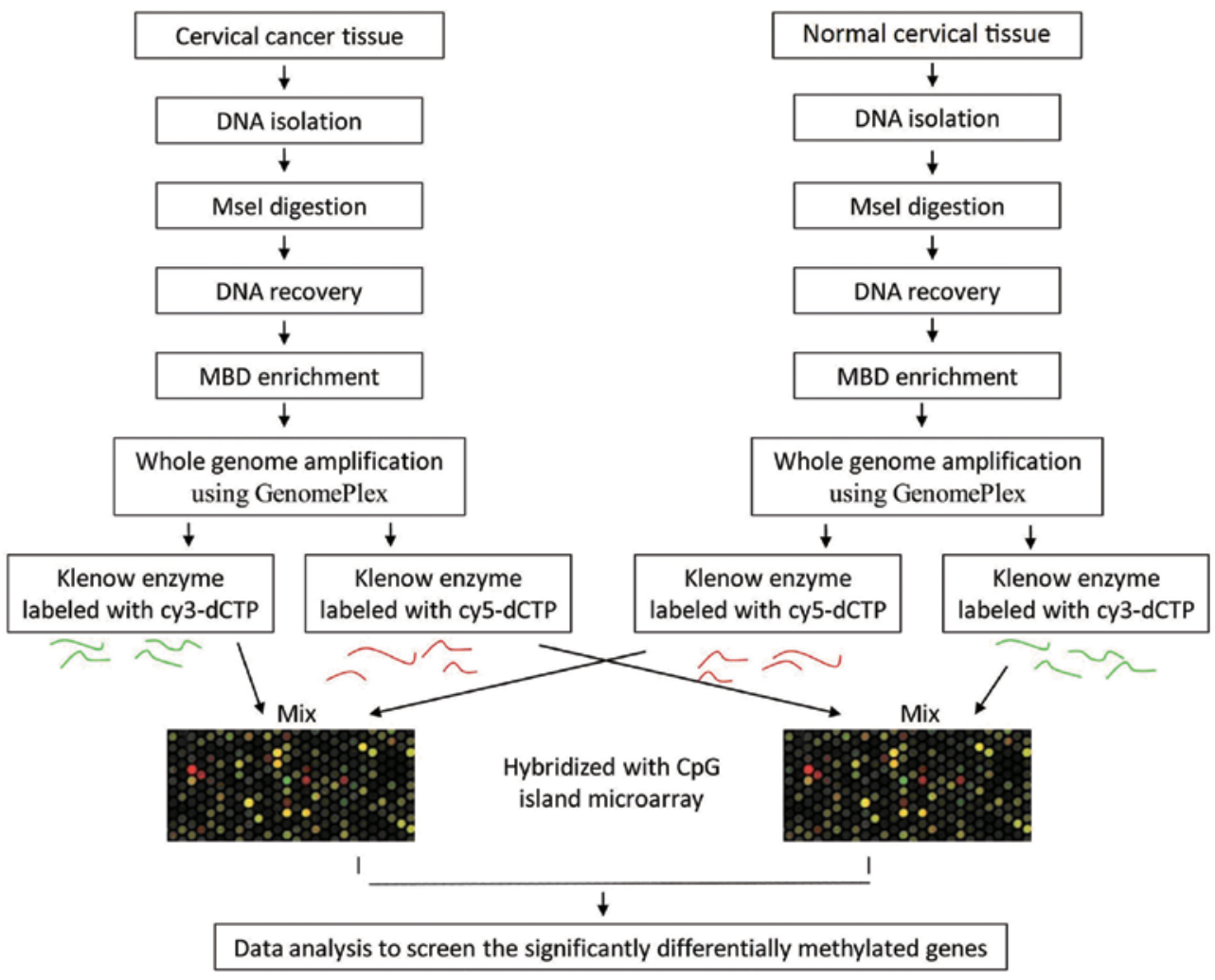

Figure 1. Schematic diagram for MIRA-assisted $\mathrm{CpG}$ island microarray in this study.

Table I. Primer pairs for direct sequencing of hypermethylated genes.

\begin{tabular}{lllr}
\hline Genes & GenBank no. & \multicolumn{1}{c}{ Primers $\left(5^{\prime} \rightarrow 3^{\prime}\right)$} & Products $(\mathrm{bp})$ \\
\hline PKLR & NM_181871 & F: GGAAGTGGAGTTGGTGAAGG & 155 \\
& & R: AAATAAACCCGTCGTCAATATAAATAC & 276 \\
DLX4 & FM_138281 & F: TGTTCGGCGATTTAAGAAGAGTAA & 207 \\
& & F: TTCTCTATCACTACCAATACCTCAA & \\
SOX9 & NM_000346 & R: TTCCCCAAACCGAATCCCTTA & \\
& &
\end{tabular}

mine the DNA methylation of SOX9 quantitatively, TaqMan-based real-time PCR for SOX9 on bisulfite-treated genomic DNA from 156 cervical scrapings was conducted on the LightCycler (Roche Scientific, cat\#4887301001). The assay format has been previously described (21). Primers excluding cytosine phosphoguanine dinucleotides and TaqMan probes specific for methylated and unmethylated states were designed to target the SOX9 gene promoter region. The sequences of the methylation-dependent primers and probes were as follows: 5'-GTATTAATTATAGGAGYGGTTGG-3' (forward primer), 5'-TCTATTCCAATTCCCCAAACC-3' (reverse primer); 5'-FAM-TTCGCGATACGGGGATTTGTTT-BHQ1-3' (methylated probe), 5'-HEX-TGGGAGTTTGTGATATGGGGATT T-BHQ1-3' (unmethylated probe). The relative signals as exemplified by the cycle threshold $(\mathrm{Ct})$ value specific for the methylated state $(\mathrm{Ctm})$ and the unmethylated state $(\mathrm{Ctu})$ were used for calculation of methylation scores (methylation score = $\left.100 /\left[1+2^{\{\mathrm{Ctm}-\mathrm{Ctu}\}}\right]\right)$. The median of at least three replicated measurements was calculated for each sample and used for statistical analysis.

Data analysis. The distribution of methylation scores for the SOX9 gene for the different grades of dysplasia is illustrated with scatter plots. Since the nature of the data precludes parametric methods, comparison of methylation levels between two or more groups was carried out using the Mann-Whitney $\mathrm{U}$ test and the Kruskal-Wallis test. The receiver operating characteristic (ROC) curve and the area under the ROC curve (AUC) were used as measures of test performance to determine if SOX9 gene methylation could distinguish SCC/CIN2-3 from CIN1/normal scrapings. The P-value reflects whether the AUC is significantly different from 0.5 (a straight line from bottom left to top right corner, implying a decision rule no better than pure chance). All statistical analyses were carried out using SPSS version 16.0 software package. Statistical significance was set at $\mathrm{P}<0.05$ and all tests performed were two sided. 
Table II. Significantly hypermethylated genes identified by MIRA based microarray.

\begin{tabular}{|c|c|c|c|c|}
\hline Gene & $\begin{array}{c}\text { Changed } \\
\text { probes }\end{array}$ & $\begin{array}{c}\text { Average } \\
\text { ratio }\end{array}$ & Gene description & Position \\
\hline SKI & 12 & 2.01 & V-ski sarcoma viral oncogene homolog (avian) & Inside \\
\hline SMYD3 & 11 & 2.55 & SET and MYND domain containing 3 & Inside \\
\hline PKLR & 6 & 1.99 & Pyruvate kinase, liver and RBC & Inside \\
\hline OLIG2 & 5 & 3.13 & Oligodendrocyte lineage transcription factor 2 & Inside, downstream \\
\hline TLX1 & 5 & 3.56 & T-cell leukemia homeobox 1 & Inside, downstream \\
\hline CUL4A & 4 & 1.78 & Cullin 4A & Inside \\
\hline DLX4 & 4 & 2.38 & Distal-less homeobox 4 & Promoter \\
\hline LHX2 & 4 & 2.61 & LIM homeobox 2 & Inside, promoter \\
\hline PRKCZ & 4 & 1.74 & Protein kinase $\mathrm{C}$, zeta & Inside \\
\hline WT1 & 4 & 3.01 & Wilms tumor 1 & Inside \\
\hline BCL11B & 3 & 3.99 & B-cell CLL/lymphoma 11B (zinc finger protein) & Inside \\
\hline HOXB13 & 3 & 1.91 & Homeobox B13 & Promoter, inside \\
\hline SOX9 & 3 & 1.89 & SRY (gender determining region Y)-box 9 & Promoter \\
\hline TBX5 & 3 & 3.04 & T-box 5 & Promoter, inside \\
\hline ZMYND8 & 3 & 2.00 & Zinc finger, MYND-type containing 8 & Inside \\
\hline EFNA5 & 2 & 3.09 & Ephrin-A5 & Inside \\
\hline EN2 & 2 & 5.44 & Engrailed homeobox 2 & Promoter, downstream \\
\hline HIPK2 & 2 & 2.12 & Homeodomain interacting protein kinase 2 & Inside \\
\hline $\mathrm{KCNB} 1$ & 2 & 1.96 & $\begin{array}{l}\text { Potassium voltage-gated channel, Shab-related subfamily, } \\
\text { member } 1\end{array}$ & Inside \\
\hline LMX1A & 2 & 1.64 & LIM homeobox transcription factor $1, \alpha$ & Inside \\
\hline MAEA & 2 & 2.21 & Macrophage erythroblast attacher & Inside \\
\hline PAX6 & 2 & 2.24 & Paired box 6 & Inside \\
\hline PTPN5 & 2 & 1.76 & Protein tyrosine phosphatase, non-receptor type 5 & Promoter, inside \\
\hline RAI1 & 2 & 3.46 & Retinoic acid induced 1 & Inside \\
\hline SLC7A8 & 2 & 2.11 & Solute carrier family 7 , member 8 & Inside \\
\hline TLX2 & 2 & 2.87 & T-cell leukemia homeobox 2 & Divergent, promoter \\
\hline TLX3 & 2 & 2.69 & T-cell leukemia homeobox 3 & Downstream \\
\hline TOLLIP & 2 & 1.70 & Toll interacting protein & Inside \\
\hline ZNF135 & 2 & 2.73 & Zinc finger protein 135 & Promoter \\
\hline ZNF146 & 2 & 2.48 & Zinc finger protein 146 & Downstream \\
\hline
\end{tabular}

Several probes are present on the microarray for each gene. Significantly hypermethylated genes were defined as genes with two positive probes, whose fold-changes of signaling ratios in cervical cancer/normal cervical tissue were above $1.5(\mathrm{P}<0.01)$ using SAM software analysis.

\section{Results}

MIRA microarray analysis. To identify significantly hypermethylated genes in cervical cancer, we performed the MIRA assay in combination with genome-wide $\mathrm{CpG}$ island arrays, which were custom made for interrogating $61,982 \mathrm{CpG}$ dinucleotides. To eliminate possible dye bias, a dye swap technical replicate was performed. A total of $504 \mathrm{CpG}$ islands, corresponding to 378 genes, were identified as differentially methylated based on positive probe criteria of a 1.5 fold-change in $\mathrm{Cy} 5 / \mathrm{Cy} 3$ or $\mathrm{Cy} 3 / \mathrm{Cy} 5$ signaling ratios of the cervical cancer tissue vs. the normal cervical tissue. Among them, 30 genes were found to have at least two positive probes and were defined to be significantly hypermethylated genes (Table II). Among the 30 genes, LMX1A and ZNF135 had previously been reported as hypermethylated in cervical cancer. TLX3,
TLX2, PAX6, EN2, EFNA5, TBX5, SOX9, HOXB13, BCL11B, LHX2, DLX4, TLX1 and OLIG2 had been reported as hypermethylated in other types of cancer. This study is the first to find hypermethylation in the remaining 14 genes.

Direct bisulfite modified sequencing. To confirm methylation data obtained from array experiments, 3 of the 30 hypermethylated genes of interest were selected for further validation using bisulfite modified sequencing, which allows the determination of the precise location of $\mathrm{CpG}$ methylation and provides useful information for designing TaqMan probes and primers. Data for the bisulfite modified sequencing in the promoter regions of the SOX9 and DLX4 genes and the intragenic region of the PKLR gene are shown in Fig. 2. Single $C$ peak was observed at multiple sites of the SOX9 promoter in cervical cancer tissue and single T peak was observed at multiple sites of the SOX9 

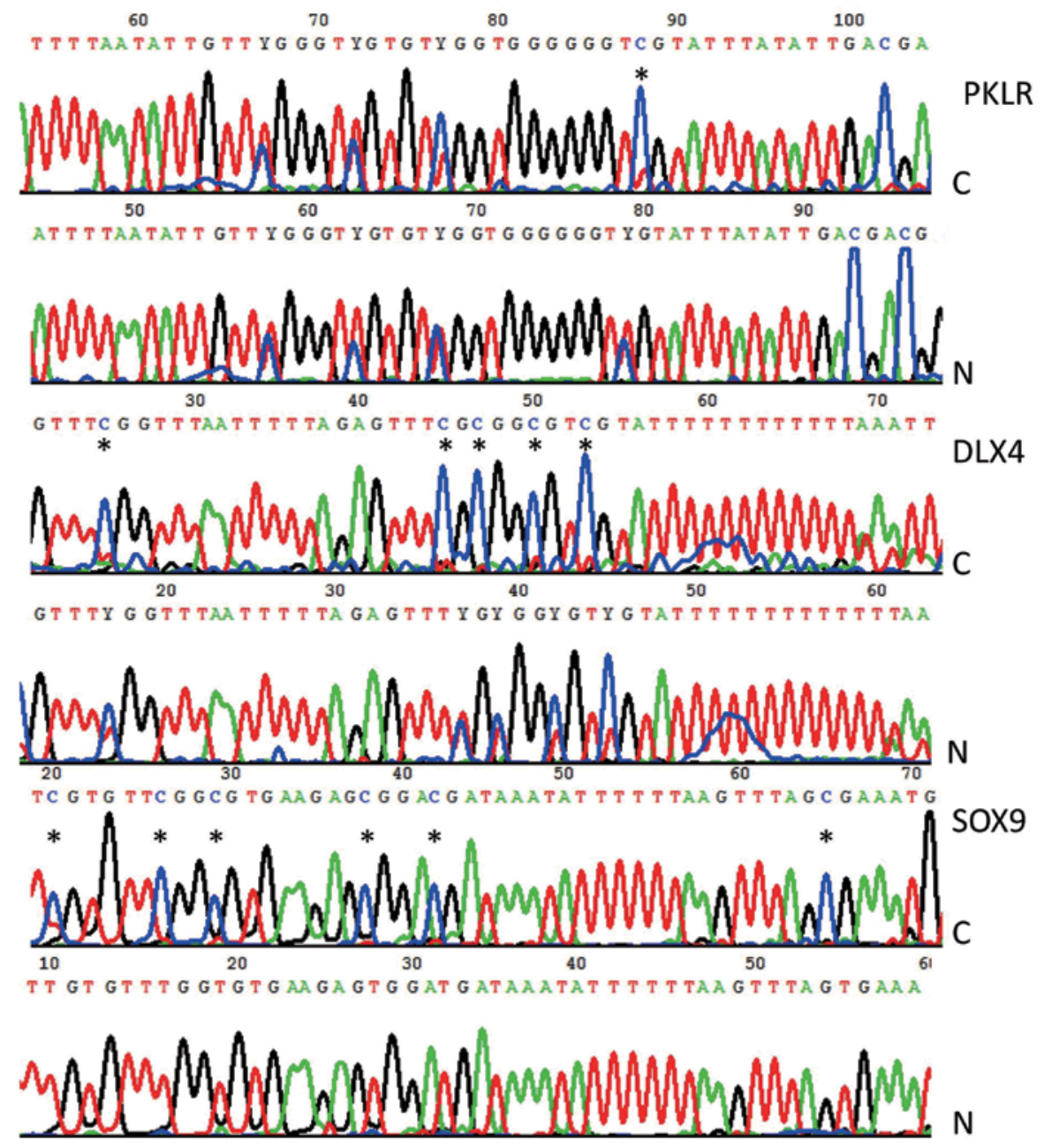

Figure 2. CpG methylations of PKLR, DLX4 and SOX9 were measured using direct bisulfite sequencing. Hypermethylated CpGs $(*)$ were observed at nucleotide +6271(88) of the PKLR gene, at nucleotide -3564(25), -3584(45), -3586(47), -3589(50) and -3592(53) of the DLX4 gene, at nucleotide -3555(20), -3561(26), $-3564(29),-3573(38),-3577(42)$ and $-3600(65)$ of the SOX9 gene in cervical cancer tissue (C), relative to normal cervical tissue (N). Y indicates T or C.

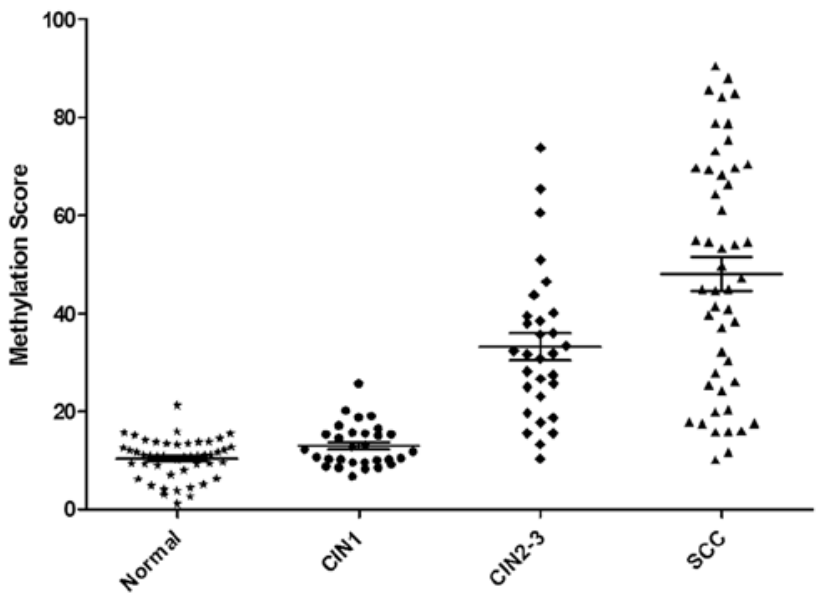

Figure 3. Plot of TaqMan-based real-time PCR showing the methylation score of the SOX9 gene in cervical scrapings. Normal, $n=48 ; C I N 1, n=30$; CIN2-3, $n=30 ;$ SCC, $n=48$. The horizontal bar indicates the mean $(\mathrm{SE})$ value for each group.

promoter in normal cervical tissue, revealing complete methylation in cervical cancer tissue and complete non-methylation in normal cervical tissue. Heterozygote $\mathrm{C} / \mathrm{T}$ double peaks were observed at multiple sites of the PKLR gene body in both cervical cancer and normal cervical tissue. Heterozygote C/T double peaks were also observed at multiple sites of the DLX4 promoter regions in normal cervical tissue; however, a single C peak was observed at multiple sites of DLX4 promoter regions in cancer tissue. These results indicate widespread methylation in cervical cancer, consistent with the microarray data and suggesting that SOX9 may have better potential as a methylation biomarker for cervical cancer detection than PKLR and DLX4.

SOX9 methylation measurement in cervical scrapings. As direct bisulfite modified sequencing indicates that SOX9 may have great potential as a methylation biomarker for cervical cancer detection, we performed TaqMan-based real-time PCR on the SOX9 gene in 156 cervical scrapings. The methylation level of the SOX9 gene increased with the severity of cervical squamous lesions (Fig. 3). The range of methylation scores for the SOX9 gene was 1.13-21.22 (mean, 10.35) in normal cervical scrapings, 1.71-25.64 (mean, 12.97) in CIN1, 10.3473.78 (mean, 33.17) in CIN2-3, and 10.10-90.50 (mean, 48.04) in SCC (Table III). The non-parametric Mann-Whitney U test was used to analyze the different methylation levels between 
Table III. The methylation score of the SOX9 gene in normal, CIN1, CIN2-3 and SCC scrapings.

\begin{tabular}{lcrrr}
\hline & $\mathrm{N}$ & Min & Max & Mean (SE) \\
\hline Normal & 48 & 1.13 & 21.22 & $10.35(0.59)$ \\
CIN1 & 30 & 6.71 & 25.64 & $12.97(0.79)$ \\
CIN2-3 & 30 & 10.34 & 73.78 & $33.17(2.77)$ \\
SCC & 48 & 10.10 & 90.50 & $48.04(3.47)$ \\
\hline
\end{tabular}

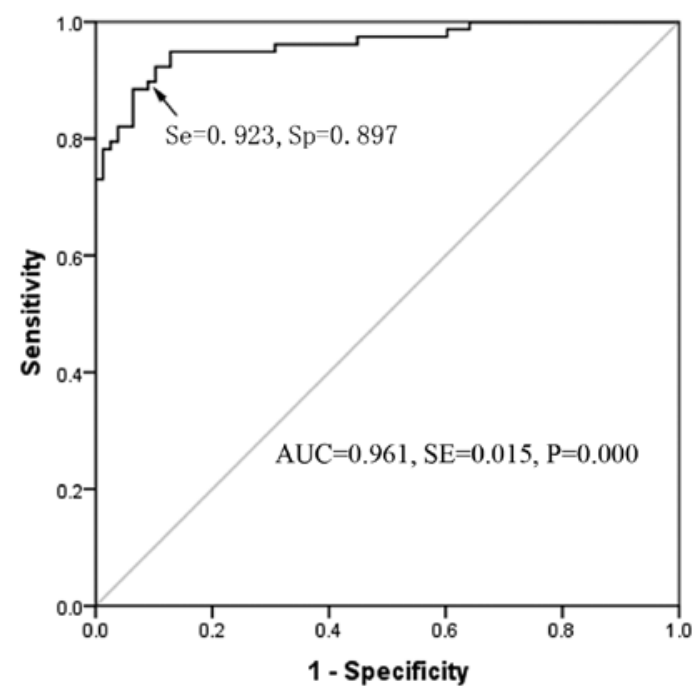

Figure 4. ROC curve based on the methylation score of the SOX9 gene in 156 cervical scrapings was generated to confirm the accuracy of diagnosis of SOX9 methylation for distinguishing SCC/CIN2-3 from CIN1/normal scrapings.

the two groups. No statistically significant difference was observed between normal and CIN1 ( $\mathrm{P}=0.062)$. However, a marked increase was observed in CIN2-3 and SCC. The difference in methylation level was statistically significant between CIN2-3 and normal ( $\mathrm{P}=0.000)$, CIN2-3 and CIN1 ( $\mathrm{P}=0.000)$, SCC and normal $(\mathrm{P}=0.000)$, SCC and CIN1 $(\mathrm{P}=0.000)$, and SCC and CIN2-3 $(\mathrm{P}=0.001)$. The methylation level of SOX9 in SCC/CIN2-3 was significantly different from CIN1/normal $(\mathrm{P}=0.000)$.

To assess for clinical application, we performed ROC analysis and calculated AUC to test the performance of SOX9 methylation on distinguishing SCC/CIN2-3 from CIN1/normal samples. The methylation score of the SOX 9 gene could be used to segregate SCC/CIN2-3 from CIN1/normal, with an AUC value of 0.961 (95\% CI, 0.933-0.990; $\mathrm{P}=0.000)$. At the optimal cut-off value a sensitivity of $92.3 \%$ and a specificity of $89.7 \%$ were obtained (Fig. 4). By using the cut-off value for the detection of SCC/CIN2-3, a methylation score of 15.76 was determined. In SCC, clinical stage and lymph node metastasis status had no effect on the methylation score (data not shown).

\section{Discussion}

The use of genome-wide approaches is accelerating the discovery of epigenetic events associated with tumorigenesis and tumor progression. In this study, we performed MIRA microarray to identify novel methylation biomarkers in cervical cancer. PKLR, DLX4 and SOX9, which have higher methylation levels but have not been associated with cervical cancer, were selected for further validation using direct bisulfite sequencing. Among these three genes, the SOX9 promoter revealed complete methylation in cervical cancer tissue and complete non-methylation in normal cervical tissue, showing it may have greater potential as a biomarker than PKLR and DLX4. So, SOX9 methylation was selected for further investigation of potential clinical application for screening cervical scrapings for degrees of precancerous lesions and malignancy. We found a progressive increase in the level of SOX9 methylation in cervical scrapings in the spectrum of cervical neoplasia and a marked increase in CIN2-3 and SCC. With a sensitivity of $92.3 \%$ and a specificity of $89.7 \%$, SOX9 methylation was able to distinguish SCC/CIN2-3 from CIN1/normal, which shows that SOX9 methylation is frequently involved in cervical carcinogenesis and may provide a valuable molecular biomarker for cervical cancer screening.

Over the last decade, several investigators have analyzed the methylation status of a variety of tumor suppressor genes and have found that DNA methylation occurs frequently in CIN3 and cervical cancer samples, but rarely in CIN1 and normal samples (22-24), suggesting that aberrant methylation may indicate an increased risk for the development of cervical cancer. Wentzensen et al (12) performed a systematic literature review of 51 published studies on the methylation status of 68 genes in cervical tissues. Among the 15 genes analyzed in detail in those studies, 7 genes (CDH1, FHIT, TERT, CDH13, MGMT, TIMP3 and HIC1) showed very heterogeneous methylation frequencies in cervical cancer, but only three markers (DAPK1, CADM1 and RAR $\beta$ ) consistently showed elevated methylation in cervical cancers across those studies. Therefore, the authors concluded that, at present, no single methylation marker can adequately perform as a cervical cancer biomarker, suggesting that a predominant methylation panel should be determined for cervical cancer screening and a high-throughput gene-wide DNA methylation profiling should be conducted to find novel methylation markers in cervical cancer. Some studies confirmed that a methylation panel could achieve high sensitivity and specificity (25-29). However, a single gene with good sensitivity, specificity and diagnostic efficacy would be more practical and cost effective than a large methylation panel (30).

The SOX family has been identified by their homology to the high-mobility group (HMG) box of sex determining region $\mathrm{Y}$, and encodes transcription factors that bind to DNA through an HMG domain and plays crucial roles in cell fate determination, differentiation and proliferation (31). SOX9, named SRY (sex determining region Y)-box 9, belongs to the subgroup of the SOX E family. SOX9 is a transcription factor that is expressed in developmental processes, including male gender determination, chondrogenesis, neurogenesis and neural crest development (32-34). In addition, SOX9 has shown tumorigenicity inhibitor effect in breast and prostate cells, suggesting a potential tumor suppressor role $(35,36)$. To the best of our knowledge, SOX9 methylation has not been previously related to cervical cancer. A high incidence of 
aberrant methylation in the promoter region of SOX9 has been described in bladder tumors, follicular lymphoma, mantle cell lymphoma, breast tumors and gastric cancer (37-41), supporting our current findings in cervical neoplasia.

Methylation is currently detected mainly by methylationsensitive PCR (MSP) or real-time fluorescent quantitative PCR techniques. MSP is suitable for the qualitative determination of methylation status as it only detects whether DNA is methylated or not (yes or no). In addition, MSP requires electrophoresis, which may cause contamination. The real-time PCR technique offers many advantages over MSP, such as higher sensitivity, better repeatability, fewer sample requirements, no electrophoresis, as well as rapid and simultaneous detections of multiple samples and/or multiple genes. However, few studies have considered that clinical cancer samples also contain non-cancer cells, such as macrophages caused by inflammation, stromal cells and vascular endothelial cells. DNA methylation levels in cancer cells are different from those in non-cancer cells. If only the methylated DNA is measured in the cancer samples, the non-cancer cells will influence the accurate quantification of the methylation in target genes. For this reason, TaqMan probes targeting methylated and non-methylated SOX9 genes were designed. Methylated SOX9 and non-methylated SOX9 were simultaneously detected in the clinical samples using two probes in the same reaction, and the percentage of methylated SOX9 was calculated. In this way, a method for quantifying SOX9 methylation was established. In this study, the methylation score of the SOX9 promoter performed well in segregating SCC/ CIN2-3 from CIN1/normal, suggesting methylation detection using real-time PCR with two TaqMan probes may have great potential for cervical cancer screening.

Since cervical scrapings are the most common specimens used for cervical cancer screening, we used cervical scrapings to validate the clinical application of SOX9 methylation. The low quantity of cervical cells in the cervical scrapings may not be of much concern as the epigenetic change may act in a field $(22,30,42)$.

Despite the promising results, this study does have limitations. HPV-DNA was not detected for the clinical specimen, so we were not able to compare its efficacy with that of the currently available Hybrid Capture 2 (HC2) HPV test. The cut-off value for SOX9 methylation was based on a hospitalbased, retrospective case-control study from a research platform, which may not be directly applicable to clinical settings from wider populations. In particular, prospective clinical trials are needed to determine whether the SOX9 methylation score can accurately predict disease progression or regression.

In summary, we have identified SOX9, a novel methylation marker that is associated with cervical carcinogenesis. We have also confirmed that the methylation level of SOX9 increases with the increase in the severity of neoplasia: SOX9 methylation can be used to distinguish SCC/CIN2-3 from CIN1/normal. Therefore, SOX9 methylation analysis may complement the current diagnostic workflow and improve early detection of cervical cancer. Before translating SOX9 methylation into a practical biomarker for cervical cancer screening, more prospective population-based epidemiologic studies are warranted.

\section{Acknowledgements}

This study was financially supported by the Beijing Municipal Education Commission (KM201010025022) and Beijing Municipal Science and Technology Commission (D09050703560905).

\section{References}

1. Parkin DM, Pisani P and Ferlay J: Estimates of the worldwide incidence of 25 major cancers in 1990. Int J Cancer 80: 827-841, 1999.

2. Kamangar F, Dores GM and Anderson WF: Patterns of cancer incidence, mortality, and prevalence across five continents: defining priorities to reduce cancer disparities in different geographic regions of the world. J Clin Oncol 24: 2137-2150, 2006.

3. Arbyn M, Bergeron C, Klinkhamer P, Martin-Hirsch P, Siebers AG and Bulten J: Liquid compared with conventional cervical cytology: a systematic review and meta-analysis. Obstet Gynecol 111: 167-177, 2008.

4. Mayrand MH, Duarte-Franco E, Rodrigues I, et al: Human papillomavirus DNA versus Papanicolaou screening tests for cervical cancer. N Engl J Med 357: 1579-1588, 2007.

5. Cuzick J, Szarewski A, Cubie H, et al: Management of women who test positive for high-risk types of human papillomavirus: the HART study. Lancet 362: 1871-1876, 2003.

6. Wright TJ, Massad LS, Dunton CJ, Spitzer M, Wilkinson EJ and Solomon D: 2006 consensus guidelines for the management of women with cervical intraepithelial neoplasia or adenocarcinoma in situ. Am J Obstet Gynecol 197: 340-345, 2007.

7. Castle PE, Schiffman M, Wheeler CM and Solomon D: Evidence for frequent regression of cervical intraepithelial neoplasiagrade 2. Obstet Gynecol 113: 18-25, 2009.

8. McCredie MR, Sharples KJ, Paul C, et al: Natural history of cervical neoplasia and risk of invasive cancer in women with cervical intraepithelial neoplasia 3: a retrospective cohort study. Lancet Oncol 9: 425-434, 2008.

9. Pretorius RG, Peterson P, Azizi F and Burchette RJ: Subsequent risk and presentation of cervical intraepithelial neoplasia (CIN) 3 or cancer after a colposcopic diagnosis of CIN 1 or less. Am J Obstet Gynecol 195: 1260-1265, 2006.

10. Esteller M, Corn PG, Baylin SB and Herman JG: A gene hypermethylation profile of human cancer. Cancer Res 61: 3225-3229, 2001.

11. Shivapurkar N, Sherman ME, Stastny V, et al: Evaluation of candidate methylation markers to detect cervical neoplasia. Gynecol Oncol 107: 549-553, 2007.

12. Wentzensen N, Sherman ME, Schiffman M and Wang SS: Utility of methylation markers in cervical cancer early detection: appraisal of the state-of-the-science. Gynecol Oncol 112: 293-299, 2009.

13. Iliopoulos D, Oikonomou P, Messinis I and Tsezou A: Correlation of promoter hypermethylation in hTERT, DAPK and MGMT genes with cervical oncogenesis progression. Oncol Rep 22: 199-204, 2009.

14. Feng Q, Balasubramanian A, Hawes SE, et al: Detection of hypermethylated genes in women with and without cervical neoplasia. J Natl Cancer Inst 97: 273-282, 2005.

15. Wisman GB, Nijhuis ER, Hoque MO, et al: Assessment of gene promoter hypermethylation for detection of cervical neoplasia. Int J Cancer 119: 1908-1914, 2006.

16. Jung S, Yi L, Jeong D, et al: The role of ADCYAP1, adenylate cyclase activating polypeptide 1 , as a methylation biomarker for the early detection of cervical cancer. Oncol Rep 25: 245-252, 2011.

17. Lai HC, Lin YW, Huang TH, et al: Identification of novel DNA methylation markers in cervical cancer. Int J Cancer 123: 161-167, 2008.

18. Sova P, Feng Q, Geiss G, et al: Discovery of novel methylation biomarkers in cervical carcinoma by global demethylation and microarray analysis. Cancer Epidemiol Biomarkers Prev 15: 114-123, 2006

19. Wang SS, Smiraglia DJ, Wu YZ, et al: Identification of novel methylation markers in cervical cancer using restriction landmark genomic scanning. Cancer Res 68: 2489-2497, 2008. 
20. Rauch TA and Pfeifer GP: The MIRA method for DNA methylation analysis. Methods Mol Biol 507: 65-75, 2009.

21. Martens JW, Nimmrich I, Koenig T, et al: Association of DNA methylation of phosphoserine aminotransferase with response to endocrine therapy in patients with recurrent breast cancer. Cancer Res 65: 4101-4117, 2005.

22. Huang TH, Lai HC, Liu HW, et al: Quantitative analysis of methylation status of the PAX1 gene for detection of cervical cancer. Int J Gynecol Cancer 20: 513-519, 2010.

23. Missaoui N, Hmissa S, Dante R and Frappart L: Global DNA methylation in precancerous and cancerous lesions of the uterine cervix. Asian Pac J Cancer Prev 11: 1741-1744, 2010.

24. Nephew KP: What will it take to obtain DNA methylation markers for early cervical cancer detection? Gynecol Oncol 112: 291-292, 2009.

25. Apostolidou S, Hadwin R, Burnell M, et al: DNA methylation analysis in liquid-based cytology for cervical cancer screening. Int J Cancer 125: 2995-3002, 2009.

26. Kim JH, Choi YD, Lee JS, Lee JH, Nam JH and Choi C: Assessment of DNA methylation for the detection of cervical neoplasia in liquid-based cytology specimens. Gynecol Oncol 116: 99-104, 2010

27. Lim EH, Ng SL, Li JL, et al: Cervical dysplasia: assessing methylation status (Methylight) of CCNA1, DAPK1, HS3ST2, PAX1 and TFPI2 to improve diagnostic accuracy. Gynecol Oncol 119: 225-231, 2010

28. Eijsink JJ, Lendvai A, Deregowski V, et al: A four-gene methylation marker panel as triage test in high-risk human papillomavirus positive patients. Int J Cancer 130: 1861-1869, 2012.

29. Eijsink JJ, Yang N, Lendvai A, et al: Detection of cervical neoplasia by DNA methylation analysis in cervico-vaginal lavages, a feasibility study. Gynecol Oncol 120: 280-283, 2011.

30. Glockner SC, Dhir M, Yi JM, et al: Methylation of TFPI2 in stoo DNA: a potential novel biomarker for the detection of colorectal cancer. Cancer Res 69: 4691-4699, 2009.

31. Lefebvre V, Dumitriu B, Penzo-Mendez A, Han Y and Pallavi B Control of cell fate and differentiation by Sry-related highmobility-group box (Sox) transcription factors. Int J Biochem Cell Biol 39: 2195-2214, 2007.
32. Kent J, Wheatley SC, Andrews JE, Sinclair AH and Koopman P: A male-specific role for SOX 9 in vertebrate sex determination. Development 122: 2813-2822, 1996.

33. Soderstrom M, Bohling T, Ekfors T, Nelimarkka L, Aro HT and Vuorio E: Molecular profiling of human chondrosarcomas for matrix production and cancer markers. Int J Cancer 100: 144-151, 2002.

34. Stolt CC, Lommes P, Sock E, Chaboissier MC, Schedl A and Wegner M: The Sox9 transcription factor determines glial fate choice in the developing spinal cord. Genes Dev 17: 1677-1689, 2003.

35. Afonja O, Raaka BM, Huang A, et al: RAR agonists stimulate SOX9 gene expression in breast cancer cell lines: evidence for a role in retinoid-mediated growth inhibition. Oncogene 21: 7850-7860, 2002

36. Drivdahl R, Haugk KH, Sprenger CC, Nelson PS, Tennant MK and Plymate SR: Suppression of growth and tumorigenicity in the prostate tumor cell line M12 by overexpression of the transcription factor SOX9. Oncogene 23: 4584-4593, 2004.

37. Enjuanes A, Fernandez V, Hernandez L, et al: Identification of methylated genes associated with aggressive clinicopathological features in mantle cell lymphoma. PLoS One 6: e19736, 2011

38. Aleman A, Adrien L, Lopez-Serra L, et al: Identification of DNA hypermethylation of SOX9 in association with bladder cancer progression using CpG microarrays. Br J Cancer 98: 466-473, 2008.

39. Bennett LB, Schnabel JL, Kelchen JM, et al: DNA hypermethylation accompanied by transcriptional repression in follicular lymphoma. Genes Chromosomes Cancer 48: 828-841, 2009.

40. Chakravarty G, Moroz K, Makridakis NM, et al: Prognostic significance of cytoplasmic SOX9 in invasive ductal carcinoma and metastatic breast cancer. Exp Biol Med 236: 145-155, 2011.

41. Sun M, Uozaki H, Hino R, et al: SOX9 expression and its methylation status in gastric cancer. Virchows Arch 460: 271-279, 2012.

42. Wang KH, Liu HW, Lin SR, Ding DC and Chu TY: Field methylation silencing of the protocadherin 10 gene in cervical carcinogenesis as a potential specific diagnostic test from cervical scrapings. Cancer Sci 100: 2175-2180, 2009. 